\title{
Antidepressants Fluoxetine Mediates Endoplasmic Reticulum Stress and Autophagy of Non-Small Cell Lung Cancer Cells Through ATF4-AKT-mTOR Signaling Pathway.
}

\section{Shali Shao}

Jinshan Hospital of Fudan University

Yajing Du

Jinshan Hospital of Fudan University

Xibing Zhuang

Jinshan Hospital of Fudan University

Tiankui Qiao ( $\square$ qiaotiankui@fudan.edu.cn )

Jinshan Hospital of Fudan University

\section{Research Article}

Keywords: Antidepressants, fluoxetine, autophagy, cancer cells

Posted Date: September 22nd, 2021

DOI: https://doi.org/10.21203/rs.3.rs-713594/v2

License: (c) (i) This work is licensed under a Creative Commons Attribution 4.0 International License.

Read Full License 
Antidepressants fluoxetine mediates endoplasmic reticulum stress and autophagy of Non-small cell lung cancer cells through ATF4-AKT-mTOR signaling pathway.

Shali Shao1, Yajing Du1, Xibing Zhuang1, Tiankui Qiao1*

${ }^{1}$ Center for Tumor Diagnosis and Therapy, Jinshan Hospital, Fudan University, Shanghai, China

Shali Shao email: 1821127008@,fudan.edu.cn

Correspondence to Prof. Tiankui Qiao, Center for Tumor Diagnosis and Therapy, Jinshan Hospital, Fudan University, Jinshan District, Shanghai 201508, China

Tel: +8618930778786

E-mail: qiaotiankui@fudan.edu.cn;

\section{Abstract:}

Cancer patients often suffer the depression with worse quality of life and prognosis. Therefore, studying how antidepressants affect the cancer is of great significance. Recent years witnessed the reports that antidepressants have the antiproliferation effect, However, in lung cancer it's still unclear about the specific molecular target. This study mainly focused on how fluoxetine influenced different types of cells and discovered the molecular basis of its inhibitory effect in lung cancer. The specific antiproliferation effect and autophagy induced by fluoxetine on lung cancer cell were shown in CCK8 and immunofluorescence. The RNA sequence hinted that the endoplasmic reticulum (ER) stress-related protein and mTOR pathway were enriched after fluoxetine treating. Western blot results revealed that the ER stress pathway was activated by fluoxetine, including PERK, ATF4, and CHOP, while the AKT/mTOR pathway was inhibited. In addition, the transfection of ATF4 siRNA further discovered that ER stress participated in the inhibition of AKT/mTOR pathway and the induction of antiproliferation and autophagy in the fluoxetine-treated cells. More importantly, fluoxetine was demonstrated to play cytotoxic activity in cancer cells without affecting normal cells. Our results showed that fluoxetine triggered the ATF4-AKT-mTOR signaling pathway to induce cell cycle arrest and autophagy restraining cancer cells' growth in lung cancer. This study found fluoxetine unaffected the proliferation of normal lung epithelial cells, providing safe clinical therapeutic strategies for lung cancer patients with depression Introduction:

Lung cancer is a highly prevalent cancer worldwide with severe lethality, accounting for $30 \%$ of cancer-related death in China ${ }^{1}$. Cancer patients suffer a high probability of getting depression, whose incidence is approximately $15 \%{ }^{2}$. And to make the situation worse, the prevalence of depression in lung cancer, ranging from $16 \%$ to $29 \% 0^{3,4}$, is larger than the average of other cancers. The status of cancer will influence the process of depression and be influenced by the depression in turn. Specifically, long-lasting suffering and unhelpful effectiveness of anticancer treatment will boost depression and eventually speed up patients' death ${ }^{5}$. Meanwhile, the influence of depression is multifaceted. Depression in cancer will accelerate the dysfunctional activation of the hypothalamic-pituitary axis; oxidative stress and inflammation; and a weakened immunosurveillance ${ }^{6}$. Thus, therapeutical approaches that can simultaneously treat cancer and depression are valuable in clinic.

Antidepressants are widely used to cure depression. In the recent years, the tricycle antidepressants (TCA) were replaced by the newer generation antidepressants, because of the serious side effects. Fluoxetine, a selective serotonin reuptake inhibitor (SSRI), is one of the latest clinical antidepressants ${ }^{7}$. Reports demonstrated that fluoxetine also had an inhibitory effect on cancer progression in ovarian cancer $^{8}$, colon cancer, and breast cancer ${ }^{9}$. However, in lung cancer what the role that fluoxetine plays in cell death is still ambiguous. 
The endoplasmic reticulum (ER) is an organelle that involves protein folding, calcium storage, and biosynthesis ${ }^{10}$. ER has a robust hemostasis system, but various factors can still destroy the balance, such as radiotherapy and chemotherapy. Under the stressed condition, the accumulation of misfolded or unfolded protein exceeds the ER protein folding capacity threshold, which will trigger ER stress ${ }^{11}$. The ER stress can activate the HSP70-type BiP/GRP78, which dissociates from luminal domains to activate three related sensors ${ }^{12}$. PKR-like ER kinase (PERK), inositol-requiring enzyme $1 \alpha$ (IRE1 $\alpha$ ), and activating transcription factor $6 \alpha(\mathrm{ATF} 6 \alpha)$ are the main intraluminal ER proteins, and the activation of these proteins can trigger Unfolded Protein Responses (UPR) to maintain the homeostasis of ER ${ }^{11}$. The role of ER stress is dual, either triggering cell death or cell survival, depending on the stress conditions and cell types ${ }^{13,14}$. When the activation of UPR fails to cope with unfolded proteins to remain ER stability, it will trigger cell apoptosis, and in different malignancies, it can also cause autophagy to re-use the organelles ${ }^{15}$. Accumulating evidences suggested that both ER stress and UPR are related to pathological processes, like cancer and depression ${ }^{16}$.

Here, we explore the anti-tumor potential of fluoxetine in lung cancer cells in vitro. We find that fluoxetine exerts an inhibitory role on cancer cells growth by inducing cell cycle arrest and autophagy without influencing normal cells. In addition, the ER stress-related pathway is involved in the anticancer treatment of fluoxetine. These results provide a new strategy of fluoxetine in the treatment of lung cancer patients with depression.

\section{Results:}

the comparison of antiproliferation effect between paroxetine and fluoxetine in multiple cell lines.

To explore the beneficial therapeutic strategies for cancer patients with depression, we used antidepressants as a breakthrough to evaluate the therapeutic potential of different drugs. The antiproliferative activities of paroxetine and fluoxetine in multiple cell types were assessed by CCK8. For NSCLC (Non-small cell lung cancer) cell lines (H460 and A549), the results showed that both paroxetine and fluoxetine inhibited the cell viability in a concentration-dependent way (Fig. 1A, B, F, G). Then, we compared the cytotoxicity of drugs in the human hepatoma cell line (Huh7). The data showed that paroxetine inhibited the growth of Huh7, while fluoxetine had a slightly inhibitory effect (Fig. 1D, I). The results of normal lung epithelial cells (BEAS-2B) and normal liver cell line (L02) indicated that paroxetine had a greater effect on normal cells than fluoxetine (Fig. 1C, E, H, J). Based on these data, we thought that the protective effect of fluoxetine on normal cells made it safer in clinical therapies, although the inhibitory effect of fluoxetine on tumor cells was not as significant as that of paroxetine, so we chose fluoxetine for further study.

\section{Fluoxetine arrested cell cycle at G0/G1.}

To clarify the inhibitory mechanism of fluoxetine, we then focused on the cell cycle. Flow cytometry was used to evaluate the distribution of the cell cycle. We observed that the percentage of the G0/G1 phase was increased with the drug concentration, these results indicated that fluoxetine could arrest the cell cycle in G0/G1 phase, and this ability was in a concentration-dependent manner (Fig. 2A, B). Meanwhile, we used western blot to evaluate the expression of cyclin-dependent kinases (CDKs) and p21, p27, the proteins related to the G1 phase. CDKs are the main regulator of the cell cycle, and CDK2 is an essential kinase for the G1/S transition ${ }^{17}$. p2 1 and p27 are well-known inhibitors of CDK2 ${ }^{18}$, which can cause cell cycle arrest at the G0/G1 phase. Our research found that fluoxetine could increase the expression of p21 and p27 and decrease CDK2 in a dose-dependent manner both in the H460 cells and 
A549 cells (Fig. 2C, D). These results were consistent with the flow cytometry analysis. Therefore, we speculated that fluoxetine might arrest the cell cycle. Altogether, the fluoxetine induced inhibitory influence on cell proliferation was demonstrated from these results.

\section{Fluoxetine induced autophagy in a dose and time-dependent way.}

Then, we shone a light on autophagy to discover the underlying mechanism of fluoxetine in regressing cell proliferation. Immunofluorescence was used to observe the autophagosome. We found that the intensity of the fluorescence increased with the drug concentration. The same phenomena could be detected in A549 cells as well (Fig. 3A). Then, the western blot was used to measure the expression of autophagy-related proteins. p62 and LC3 are the two markers of autophagy; therefore, these proteins can represent autophagy to some extent. We found that as the drug concentration increased, the expression of p62 and LC3B continued to increase, while the level of LC3A decreased (Fig. 3B). Another obvious phenomenon that could be observed was that the induction of autophagy flux gradually became more significant over time. Compared with control group, the level of LC3B changed from $3 \mathrm{~h}$ and gradually became obvious, reaching the maximum at $24 \mathrm{~h}$ (Fig. 3C, D). Therefore, we thought autophagy could be induced by fluoxetine in a dose-dependent and time-dependent fashion.

\section{Fluoxetine had a connection with ER stress and the mTOR signaling pathway.}

In order to investigate the specific mechanism of fluoxetine, we chose $\mathrm{H} 460$ cells that were more sensitive to the drug. RNA sequencing (RNA-seq) was used to study the transcriptome of cells treated with fluoxetine and analyze the difference with control treatment. In this result, 166 differentially expressed genes $(\mathrm{P}<0.05$, fold change $\geq 2)$ were identified, of which 12 genes were downregulated, and 154 genes were upregulated (Fig. 4A, B). We further analyzed the autophagy-related genes in the gene pool, and four genes proved to be significantly different. Interestingly, we found DDIT3, also known as C/EBP homologous protein (CHOP), was upregulated after fluoxetine treatment (Fig. 4C). CHOP is a characteristic biomarker of ER stress ${ }^{19}$. A plethora of studies has been conducted to investigate the relationship between ER stress and autophagy ${ }^{20}$. Taking these into consideration, we wanted to explore the connection between autophagy and ER stress after fluoxetine treatment. Western blot was used to detect the ER stress-related markers. BIP expression was detected firstly, and it was found that fluoxetine induced an up-regulation of BIP levels. The activation of BIP can trigger the downstream sensors and the PERK pathway involved in autophagy is one of these sensors ${ }^{21}$. The result of western blot showed that fluoxetine increased the content of PERK, activating transcription factor 4 (ATF4), and CHOP in a dosedependent way (Fig. 4E). All these results indicated that fluoxetine had a close relationship with ER stress.

The analysis of the Kyoto Encyclopedia of Genes and Genomes (KEGG) showed that the genes were enriched in 8 pathways which included biosynthesis, metabolism, mechanistic target of rapamycin kinase (mTOR) signaling pathway, Lysosome, and AMPK signaling pathway (Fig. 4D). AKT serine/threonine kinase (AKT)/mTOR signaling pathway has been proved to play a critical role in regulating the process of autophagy ${ }^{22}$. Hence, we detected the expression of AKT and mTOR. In our results, the fluoxetine could down-regulate the p-AKT/AKT and p-mTOR/mTOR in a dose-dependent way (Fig. 4F). Based on these results, we thought that the ER stress and AKT/mTOR signaling pathway might play a significant role in fluoxetine-treated cells.

\section{AKT/mTOR signaling pathway was regulated through ATF4 in fluoxetine treatment.}


A recent study has demonstrated that ER stress can regulate the mTOR signaling pathway to play a role $^{23}$. Thus, we further investigated the regulation of ER stress and AKT/mTOR signaling pathway induced by fluoxetine. The role of CHOP and ATF4 were identified firstly. The analysis of bioinformatics showed that ATF4 had a higher expression in lung cancer tissues (Fig. 5A), and the higher expression of ATF4 was significantly related to longer overall survival (OS) and disease-specific survival (DSS) of NSCLC patients (Fig. 5B, C). The role of CHOP in expression and prognosis is not obvious (data not shown). Therefore, we chose ATF4 for further experimental validation. To clarify whether the downregulation of AKT/mTOR signaling pathway was induced by ATF4, the cells were transfected with ATF4 siRNA and the western blot was used to examine the efficiency of siRNA. We next investigated whether fluoxetine-mediated inhibition of AKT/mTOR occurred through increased ATF4. The cells were transfected with ATF4 siRNA, and levels of p-AKT/AKT and p-mTOR/mTOR were assessed after fluoxetine treatment. Compared with NC-siRNA, the expression of $\mathrm{p}-\mathrm{AKT} / \mathrm{AKT}$ and $\mathrm{p}-\mathrm{mTOR} / \mathrm{mTOR}$ recovered in ATF4-knockdown cells, and the expression was reduced after fluoxetine treatment (Fig. 5D).

\section{The fluoxetine-induced anticancer effect through the ATF4-AKT-mTOR signaling pathway.}

And then to analyze whether the fluoxetine induced anticancer effect through the ATF4-AKTmTOR signaling pathway. Cell proliferation, cell cycle, and autophagy were measured after ATF4 siRNA treatment. The CCK8 assay showed that the cell viability recovered after ATF4 knockdown (Fig. 6A). The analysis of cell cycle results found that the proportion of G0/G1 phase and the expression of G0/G1 related proteins was reduced after ATF4 knockdown (Fig. 6B, C, E). Moreover, the results of immunofluorescence and protein level of LC3B further confirmed that the treatment with ATF4 siRNA decreased the induction of autophagy after fluoxetine treatment (Fig. 6D, E). All these data indicated that the ATF4-AKT-mTOR signaling pathway exerted a significant role in the fluoxetine-induced anticancer effect.

\section{Fluoxetine exerted anti-tumor effects while not damaging normal cells.}

The previous result showed that fluoxetine had no inhibitory effect on normal lung epithelial cells. To confirm this result further, we used flow cytometry and immunofluorescence to analyze. The results demonstrated that fluoxetine was unacted on the cell cycle and autophagy (Fig. 7A, B, C). Western blot also revealed that the protein levels of cell cycle and autophagy were slightly changed after fluoxetine treatment (Fig. 7D, E).

\section{Discussion:}

Depression is frequently found in the cancer patients, with higher prevalence than general population ${ }^{24}$. Antidepressants are the common drugs to cure depression and also have the anticancer effect $^{25}$. However, different antidepressants may have different impact on tumors. In our research, we discovered that fluoxetine inhibited the cell proliferation and induced autophagy of lung cancer cells H460 and A549 cells. Compared with paroxetine, we found that fluoxetine specifically inhibited the proliferation of H460 and A549 cells without influencing liver cancer cell Huh7. Then, we found that fluoxetine upregulated the proportion of G0/G1 phase and affected cell cycle-related proteins, which were consistent with the previous findings ${ }^{26,27}$.

In general, the existing anticancer drugs not only target at cancer cells but also normal cells, which will cause serious side effects, including hair loss, neurotoxicity, and cardiotoxicity ${ }^{28}$. Therefore, developing ideal anticancer drugs that specifically target at cancer cells is of great significance. More 
interestingly and importantly, we first found that fluoxetine hardly affected BEAS-2B. In our results, fluoxetine has less toxicity in normal lung epithelial cell.

Autophagy is a conserved catabolic process that is induced by stresses and cellular signals. The process of autophagy contains the production of double-membraned vesicles, which engulf the cellular components, forming the complex called autophagosomes ${ }^{16}$. To get further insights into the mechanisms and targets of the fluoxetine anti-tumor activity in lung cancer cells, we found that fluoxetine induced autophagy. For the first time paying attention to the autophagy induced by fluoxetine in lung cancer cells, we further detected the expression of autophagy-related proteins. P62, also called sequestosome-1 (SQSTM1), is a crucial regulator in autophagy ${ }^{29}$. p62 interacts with LC3 to form autophagosome, ultimately fusing with lysosome and degradation ${ }^{30}$. Therefore, the level of p62 can reflect the activity of lysosomes. In our study, both p62 and LC3 increased with fluoxetine concentrations. We speculated that fluoxetine could induce autophagy, but it also destroyed the function of lysosomes to a certain extent, which led to an abnormal increase of $\mathrm{p} 62$.

The result of RNA-seq hinted that the CHOP had a connection with autophagy. CHOP is a marker of UPR, and UPR is an adaptive response to ER stress. Previous studies shown that some drugs can continuously induce ER stress through the UPR pathway to exert anticancer effects, which made UPR a potential target for anti-cancer therapy ${ }^{15}$. PERK is an essential sensor for translation regulation, which increases the expression of ATF4 and CHOP during the URP ${ }^{31}$. Moreover, the PERK-ATF4 signaling pathway was found to be activated in some cancers and the PERK-induced autophagy decreased the damage of ROS accumulation ${ }^{32,33}$. And ATF4 and CHOP have also been discovered to participate in the induction of autophagy ${ }^{34}$. We analyzed the ER stress in fluoxetine-treated cells. The UPR-related proteins such as BIP, PERK, ATF4, and CHOP were detected, the levels of these proteins all increased with the increasing concentration. Previous research found that fluoxetine reversed depressive-like behavior in mice through PI3K/Akt $/ \mathrm{mTOR} / \mathrm{p}$-ERK1/2 signaling pathways ${ }^{35}$. Coupled with the result of KEGG, we wonder whether AKT/mTOR signaling pathway participated in the fluoxetine treatment. The activation of signaling pathway is a cascade reaction. PI3K phosphorylates AKT, and the activated AKT further phosphorylates mTOR. The results of western blot showed that the levels of p-AKT/AKT, p$\mathrm{mTOR} / \mathrm{mTOR}$ were significantly decreased, indicating the involvement of the AKT/mTOR pathway. Remarkably, the AKT/mTOR pathway was also related to the activation of ER stress. We now have confirmed that fluoxetine induced ER stress which increased ATF4, this upregulation further inhibited AKT/mTOR signaling pathway.

To our knowledge, there is no report on the study of the relationship between fluoxetine with ER stress and anticancer effect in lung cancer cells. So, it is of great importance to discover the involvement of the ATF4-AKT-mTOR signaling pathway in the anti-tumor activity of fluoxetine in lung cancer cells. By regulating p21 and p27, the AKT/mTOR influenced the cell cycle to change cell proliferation. In addition, mTOR has been recognized as a critical regulator of autophagy ${ }^{36}$, and the AKT/mTOR pathway has also been widely reported to be related to autophagy in neurodegeneration ${ }^{22}$ and cancer treatment ${ }^{37}$. In line with these results, we further confirmed that the fluoxetine could activate the ATF4-AKT-mTOR pathway to induce cell cycle arrest and autophagy to restraint cancer cells' growth without affecting normal cells (Fig.8).

Although it has been confirmed that fluoxetine has a potential anticancer effect, its molecular targets in certain cancer are quite unclear. Compared with previous reports, our results elaborate the molecular mechanism of fluoxetine in lung cancer. However, this study only proved the limited phenotypes induced by the ATF4-AKT-mTOR pathway after fluoxetine treatment, and the further researches need to explore 
the broader functions of fluoxetine and investigate the influence of fluoxetine on multiple cell types, such as immunocytes. And based on the different effect of fluoxetine between normal cells and cancer cells, our future work will focus on the early tumor prevention with the use of fluoxetine and explore whether timely use of fluoxetine can alleviate the development of tumors in patients with depression.

\section{Conclusion:}

In conclusion, we discovered that fluoxetine exerted an antiproliferation role in non-small lung cancer cells and induced cell cycle arrest, ER stress and autophagy by triggering ATF4-AKT-mTOR signaling pathway. More importantly, the cell-killing effect of fluoxetine had no influence on normal cells, which made it safer in the clinical therapy of lung cancer patients with depression.

\section{Methods and materials:}

\section{Cell culture and Reagents}

Fluoxetine was purchased by Sigma-Aldrich (Shanghai, China). The human lung cancer cell lines (H460 and A549) and normal lung epithelial cell (BEAS-2B) were supplied by Cell Bank (Shanghai, China). The human hepatoma cell line (Huh7) and normal liver cell line (L02) were gifted by Prof. DuoJiao Wu (Zhongshan hospital, Shanghai, China). Primary antibody including p21, p27, CDK2, p62. LC3, PERK, BIP, ATF4, CHOP, p-AKT, AKT, p-mTOR, mTOR, $\beta$-actin, and GAPDH were bought from Cell Signaling Technology (Danvers, MA, USA). The cells were cultured in complete RPMI-1640 medium (Sigma, Louis, Missouri, USA) with $10 \%$ fetal bovine serum (FBS) (Biological Industries, Israel) at $37^{\circ} \mathrm{C}$ under $5 \% \mathrm{CO}_{2}$.

\section{Cell Counting Kit-8 (CCK-8) assay}

The cells were seeded in the 96 -well plates (5000 cells/well). After 24 hours of incubation, the cells were treated with fluoxetine for $24 \mathrm{~h}$. Then, add 10ul/well CCK8 solution (Do jindo, Laboratories, Kumamoto, Japan) after changing the medium to FBS-free RPMI-1640 in the dark room. The cells were incubated at $37^{\circ} \mathrm{C}$ for 1 hour. The OD value was gathered at $450 \mathrm{~nm}$.

\section{Analysis of cell cycle}

The cells were seeded in the 6 -well plates $\left(20 \times 10^{4}\right.$ cells/well). After 24 hours of incubation, the cells were treated with the corresponding concentration of a drug. 24 hours later, the cells were trypsinized and washed with PBS, and then, the cells were suspended with $70 \%$ ethyl alcohol at $-20^{\circ} \mathrm{C}$. The next day, the cells were stained with PI/RNase Staining Buffer (BD Bioscience) for 15 minutes after discarding the ethyl alcohol. Flow cytometer (Beckman Coulter) and ModFit LT 5.0 software were used to collect and analyze the results, respectively.

\section{Immunofluorescence (IF)}

The cells were seeded in the 96 -well plates ( 5000 cells/well). After 24 hours of incubation, the cells were treated with the corresponding concentration of the drug for the next 24 hours. After discarding the solution, the cells were fixed with $4 \%$ paraformaldehyde, then, the cells were permeabilized with $0.1 \%$ Triton X-100 and blocked with 5\% BSA. The cells were incubated with primary antibody at $4{ }^{\circ} \mathrm{C}$ overnight. On the next day, the cells were incubated with fluorophore-labeled secondary antibody for 1 hour and washed with PBST, DAPI was used to stain the nucleus before observation. All of these steps were produced under dark condition.

\section{RNA-sequencing experiment}

The cells were seeded in the 6 -well plates $\left(20 \times 10^{4}\right.$ cells/well). After 24 hours of incubation, the 
cells were treated with the corresponding concentration of the drug for the next 24 hours. After trypsining and washing, the total RNA was extracted from cells using TRIzol (Invitrogen). Library construction and sequencing were performed by HaploX Genomics Center (Jiangxi, China). The libraries were sequenced on an Illumina PE150 platform.

\section{Small interfering RNA (siRNA) experiment}

The siRNA sequences against ATF4 were synthesized by Gima Company: ATF4-siRNA, 5'TCCCTCAGTGCATAAAGGA-3'. NC-siRNA, 5' - UUCUCCGAACGUGUCACG A-3'. Transfecting cells with 5ul siRNA plasmid using $8 \mathrm{ul} \mathrm{X-tremeGENE,} \mathrm{based} \mathrm{on} \mathrm{the} \mathrm{manufacturer's} \mathrm{instruction,} \mathrm{and}$ treating cells with the previous drugs after transfection

\section{Western blot}

The cells were extracted with a mixture of SDS and PMSF. The protein samples were measured with a BCA assay kit (ThermoFisher, USA). Then, SDS-PAGE gel was used to distinguish the protein, PVDF membrane to transfer protein, and 5\% milk to block protein. After that, the membrane was incubated with primary antibodies at $4^{\circ} \mathrm{C}$ overnight. On the next day, the membrane was washed with TBST and incubated with a secondary antibody. ECL (Millipore, Billerica, MA) was applied to detect the band.

\section{Statistical analysis}

Statistical differences between the compared groups were determined by one-way ANOVA analysis and Tukey's test in Graphpad Prism 7.0. Transcriptome data together with clinical characteristics were downloaded and processed with $\mathrm{R}$ version 4.0.1. Independent $\mathrm{t}$-tests were used to assess the difference of gene expression between tumor tissues and adjacent normal tissues. Kaplan-Meier curve and log-rank test were usually performed to analyze the survival probability between groups based on differences in gene expression. All values were expressed as mean \pm SD. $\mathrm{P}$ values $<0.05$ indicates statistically significant.

\section{References:}

1. Chen, W. et al. Cancer Statistics in China, 2015. CA Cancer J Clin. 66, 115-132 (2016).

2. Mitchell, A. J. et al. Prevalence of Depression, Anxiety, and Adjustment Disorder in Oncological, Haematological, and Palliative-Care Settings: A Meta-Analysis of 94 Interview-Based Studies. Lancet Oncol. 12, 160-174 (2011).

3. Hopwood, P. \& Stephens, R. J. Depression in Patients with Lung Cancer: Prevalence and Risk Factors Derived From Quality-of-Life Data. J. Clin. Oncol. 18, 893-903 (2000).

4. Walker, M. S., Zona, D. M. \& Fisher, E. B. Depressive Symptoms After Lung Cancer Surgery: Their Relation to Coping Style and Social Support. Psychooncology. 15, 684-693 (2006).

5. Akechi, T., Okuyama, T., Onishi, J., Morita, T. \& Furukawa, T. A. Psychotherapy for Depression Among Incurable Cancer Patients. Cochrane Database Syst Rev. D5537 (2008).

6. Bortolato, B. et al. Depression in Cancer: The Many Biobehavioral Pathways Driving Tumor Progression. Cancer Treat. Rev. 52, 58-70 (2017).

7. Ginsburg, A. Cancer-Related Depression and Potential Pharmacologic Therapies. Proc (Bayl Univ Med Cent). 21, 439-441 (2008).

8. Lee, C. S., Kim, Y. J., Jang, E. R., Kim, W. \& Myung, S. C. Fluoxetine Induces Apoptosis in Ovarian Carcinoma Cell Line Ovcar-3 through Reactive Oxygen Species-Dependent Activation of Nuclear Factor-Kappab. Basic Clin Pharmacol Toxicol. 106, 446-453 (2010). 
9. Stepulak, A. et al. Fluoxetine Inhibits the Extracellular Signal Regulated Kinase Pathway and Suppresses Growth of Cancer Cells. Cancer Biol. Ther. 7, 1685-1693 (2008).

10. Janssens, S., Pulendran, B. \& Lambrecht, B. N. Emerging Functions of the Unfolded Protein Response in Immunity. Nat. Immunol. 15, 910-919 (2014).

11. Bettigole, S. E. \& Glimcher, L. H. Endoplasmic Reticulum Stress in Immunity. Annu. Rev. Immunol. 33, 107-138 (2015).

12. Bertolotti, A., Zhang, Y., Hendershot, L. M., Harding, H. P. \& Ron, D. Dynamic Interaction of Bip and Er Stress Transducers in the Unfolded-Protein Response. Nat. Cell Biol. 2, 326-332 (2000).

13. Jang, J. H., Kim, Y. J., Kim, H., Kim, S. C. \& Cho, J. H. Buforin Iib Induces Endoplasmic Reticulum Stress-Mediated Apoptosis in Hela Cells. Peptides. 69, 144-149 (2015).

14. Shen, Y. et al. The Switch From Er Stress-Induced Apoptosis to Autophagy Via Ros-Mediated Jnk/P62 Signals: A Survival Mechanism in Methotrexate-Resistant Choriocarcinoma Cells. Exp. Cell Res. 334, 207-218 (2015).

15. Lin, Y., Jiang, M., Chen, W., Zhao, T. \& Wei, Y. Cancer and Er Stress: Mutual Crosstalk Between Autophagy, Oxidative Stress and Inflammatory Response. Biomed. Pharmacother. 118, 109249 (2019).

16. Lebovitz, C. B. et al. Precision Autophagy: Will the Next Wave of Selective Autophagy Markers and Specific Autophagy Inhibitors Feed Clinical Pipelines? Autophagy. 11, 1949-1952 (2015).

17. Du WW et al. Foxo3 Circular Rna Retards Cell Cycle Progression Via Forming Ternary Complexes with P21 and Cdk2. Nucleic Acids Res. 44, 2846-2858 (2016).

18. Levkau, B. et al. Cleavage of P21Cip1/Waf1 and P27Kip1 Mediates Apoptosis in Endothelial Cells through Activation of Cdk2: Role of a Caspase Cascade. Mol. Cell. 1, 553-563 (1998).

19. Senft, D. \& Ronai, Z. A. Upr, Autophagy, and Mitochondria Crosstalk Underlies the Er Stress Response. Trends Biochem. Sci. 40, 141-148 (2015).

20. Song, S., Tan, J., Miao, Y. \& Zhang, Q. Crosstalk of Er Stress-Mediated Autophagy and Er-Phagy: Involvement of Upr and the Core Autophagy Machinery. J. Cell. Physiol. 233, 3867-3874 (2018).

21. Kouroku, Y. et al. Er Stress (Perk/Eif2Alpha Phosphorylation) Mediates the Polyglutamine-Induced Lc3 Conversion, an Essential Step for Autophagy Formation. Cell Death Differ. 14, 230-239 (2007).

22. Heras-Sandoval, D., Perez-Rojas, J. M., Hernandez-Damian, J. \& Pedraza-Chaverri, J. The Role of $\mathrm{Pi} 3 \mathrm{~K} / \mathrm{Akt} / \mathrm{Mtor}$ Pathway in the Modulation of Autophagy and the Clearance of Protein Aggregates in Neurodegeneration. Cell. Signal. 26, 2694-2701 (2014).

23. Yao, W. et al. Delicaflavone Induces Ros-Mediated Apoptosis and Inhibits Pi3K/Akt/Mtor and Ras/Mek/Erk Signaling Pathways in Colorectal Cancer Cells. Biochem. Pharmacol. 171, 113680 (2020).

24. Bortolato, B. et al. Depression in Cancer: The Many Biobehavioral Pathways Driving Tumor Progression. Cancer Treat. Rev. 52, 58-70 (2017).

25. Hsu, L. C., Tu, H. F., Hsu, F. T., Yueh, P. F. \& Chiang, I. T. Beneficial Effect of Fluoxetine On AntiTumor Progression On Hepatocellular Carcinoma and Non-Small Cell Lung Cancer Bearing Animal Model. Biomed. Pharmacother. 126, 110054 (2020).

26. Marcinkute, M., Afshinjavid, S., Fatokun, A. A. \& Javid, F. A. Fluoxetine Selectively Induces P53Independent Apoptosis in Human Colorectal Cancer Cells. Eur. J. Pharmacol. 857, 172441 (2019).

27. Chen, W. T. et al. Fluoxetine Induces Apoptosis through Extrinsic/Intrinsic Pathways and Inhibits Erk/Nf-Kappab-Modulated Anti-Apoptotic and Invasive Potential in Hepatocellular Carcinoma Cells in Vitro. Int. J. Mol. Sci. 20, (2019). 
28. Singh, S., Sharma, B., Kanwar, S. S. \& Kumar, A. Lead Phytochemicals for Anticancer Drug Development. Front. Plant Sci. 7, 1667 (2016).

29. Liu, W. J. et al. P62 Links the Autophagy Pathway and the Ubiqutin-Proteasome System upon Ubiquitinated Protein Degradation. Cell. Mol. Biol. Lett. 21, 29 (2016).

30. Pankiv, S. et al. P62/Sqstm1 Binds Directly to Atg8/Lc3 to Facilitate Degradation of Ubiquitinated Protein Aggregates by Autophagy. J. Biol. Chem. 282, 24131-24145 (2007).

31. Harding, H. P., Zhang, Y., Bertolotti, A., Zeng, H. \& Ron, D. Perk is Essential for Translational Regulation and Cell Survival During the Unfolded Protein Response. Mol. Cell. 5, 897-904 (2000).

32. Atkins, C. et al. Characterization of a Novel Perk Kinase Inhibitor with Antitumor and Antiangiogenic Activity. Cancer Res. 73, 1993-2002 (2013).

33. Dey, S. et al. Atf4-Dependent Induction of Heme Oxygenase 1 Prevents Anoikis and Promotes Metastasis. J. Clin. Invest. 125, 2592-2608 (2015).

34. B'Chir, W. et al. The Eif2Alpha/Atf4 Pathway is Essential for Stress-Induced Autophagy Gene Expression. Nucleic Acids Res. 41, $7683-7699$ (2013).

35. Amin, N. et al. Optimized Integration of Fluoxetine and 7, 8-Dihydroxyflavone as an Efficient Therapy for Reversing Depressive-Like Behavior in Mice During the Perimenopausal Period. Prog Neuropsychopharmacol Biol Psychiatry. 101, 109939 (2020).

36. Shi, B., Ma, M., Zheng, Y., Pan, Y. \& Lin, X. Mtor and Beclin1: Two Key Autophagy-Related Molecules and their Roles in Myocardial Ischemia/Reperfusion Injury. J. Cell. Physiol. 234, 1256212568 (2019).

37. Xu, Z. et al. Targeting Pi3K/Akt/Mtor-Mediated Autophagy for Tumor Therapy. Appl Microbiol Biotechnol. 104, 575-587 (2020).

Acknowledgement: This work was supported by the Key Subject Construction Program of Shanghai Health Administrative Authority (Grant No. ZK2019B30)

Authors' contributions: The concept and experiments were designed by TQ and SS; the experiments was performed by SS, YD, and XZ; the manuscript draft was prepared and revised by SS and TQ. All the authors agreed the final proof.

Conflicts of interest/Competing interests: The authors declare that they have no conflict of interest

Ethics approval: All analyses were based on previous published studies; thus, no ethical approval and patient consent are required. 
A

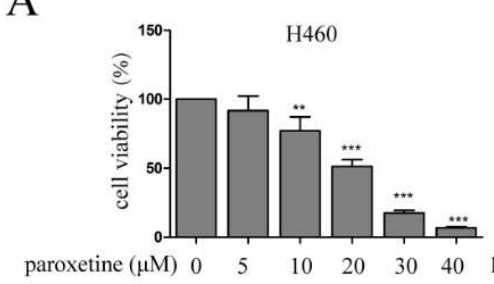

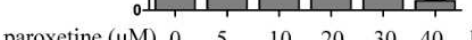

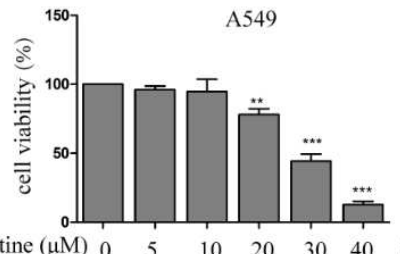

$\mathrm{C}$

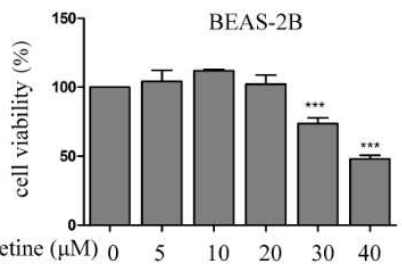

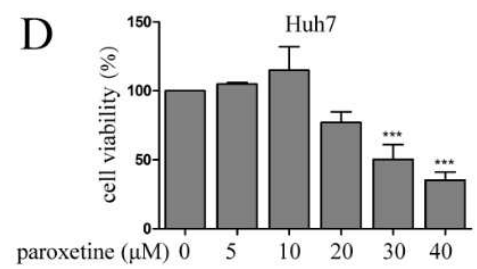

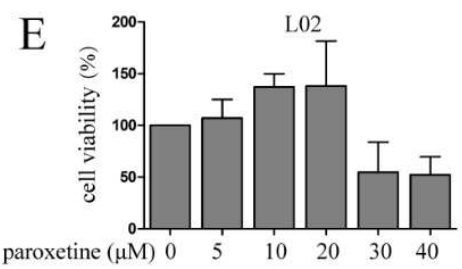

$\mathrm{F}$

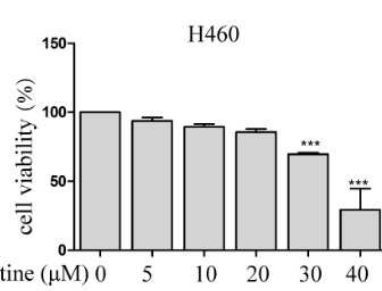

G
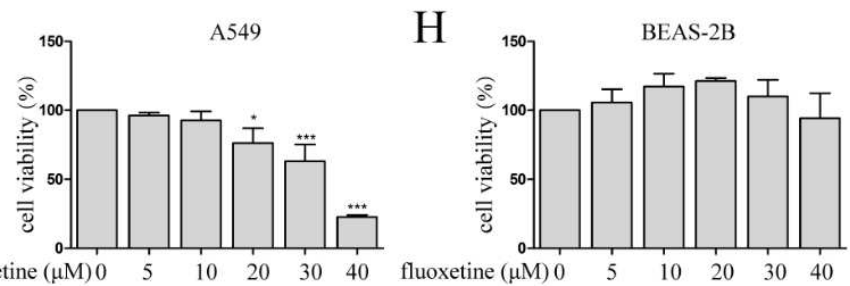

I

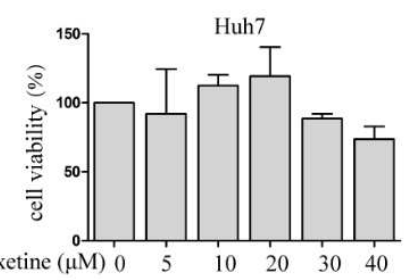

$\mathrm{J}$

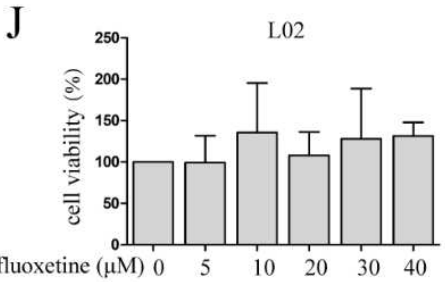

Figure.1: The comparison of paroxetine and fluoxetine for antiproliferation effect in multiple cell lines. (A-E) lung cancer cells (H460 and A549), normal lung epithelial cells (BEAS-2B), human hepatoma cell line (Huh7), and normal liver cell line (L02) were treated with paroxetine $(0,5,10,20,30$, and $40 \mu \mathrm{M}$ ) for 24h. CCK8 was used to measure cell viability. (F-J) The H460, A549, BEAS-2B, Huh7, L02 cells were treated with fluoxetine $(0,5,10,20,30$, and $40 \mu \mathrm{M})$ for $24 \mathrm{~h}, \mathrm{CCK} 8$ was used to measure cell viability. Data are presented as mean $\pm \mathrm{SD} ;{ }^{*} \mathrm{P}<0.05,{ }^{*} * \mathrm{P}<0.01,{ }^{* * *} \mathrm{P}<0.001$. 
A

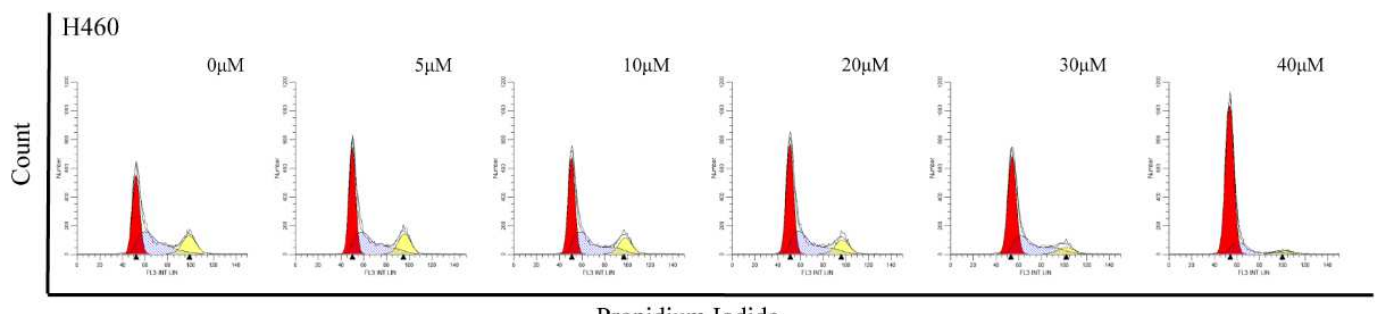

Propidium Iodide

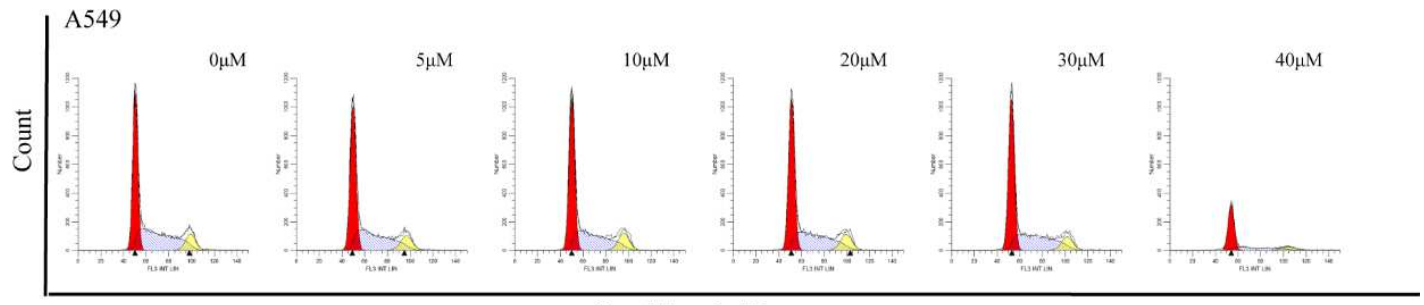

Propidium Iodide

B
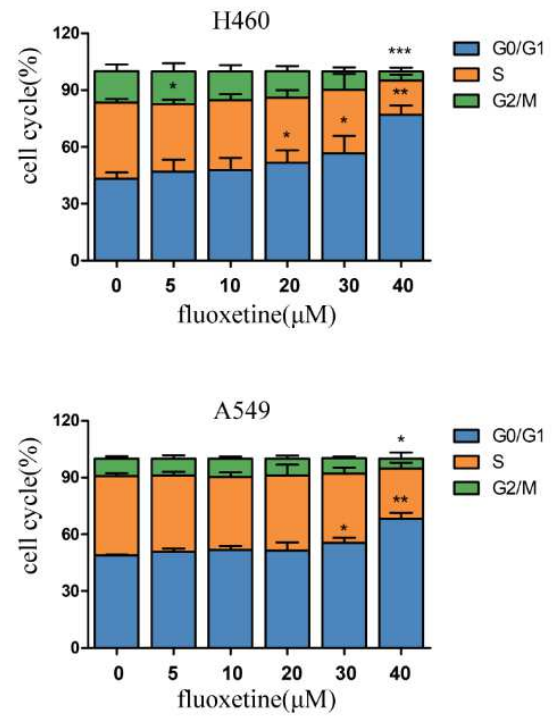

$\mathrm{D}$
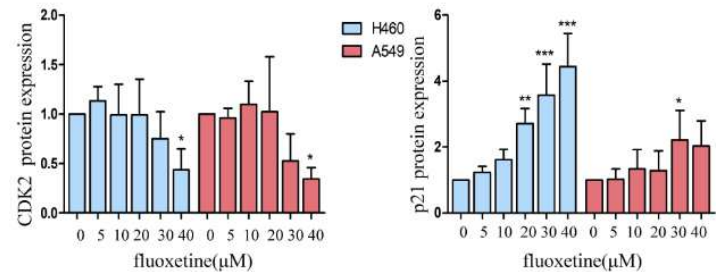

$\mathrm{C}$
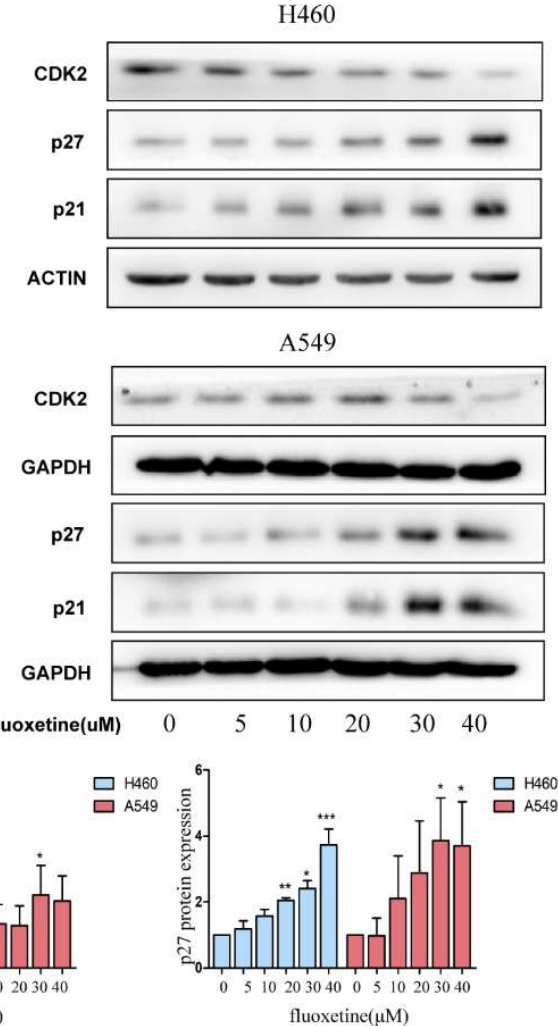

Figure.2: Fluoxetine arrested cell cycle at G0/G1. (A) The cells were treated with fluoxetine $(0-40 \mu \mathrm{M})$ for $24 \mathrm{~h}$, performed PI/RNase staining and analyzed with flow cytometry. (B) The distribution of the cell cycle was analyzed by ModFit LT 5.0 software (C) The result of the Western blot of CDK2, p27, and p21. The images were collected from different parts of the same gel. (D) Quantitative analysis of optical band densitometry. Data are presented as mean $\pm \mathrm{SD} ;{ }^{*} \mathrm{P}<0.05,{ }^{*} \mathrm{P}<0.01,{ }^{* * *} \mathrm{P}<0.001$. 
$A$
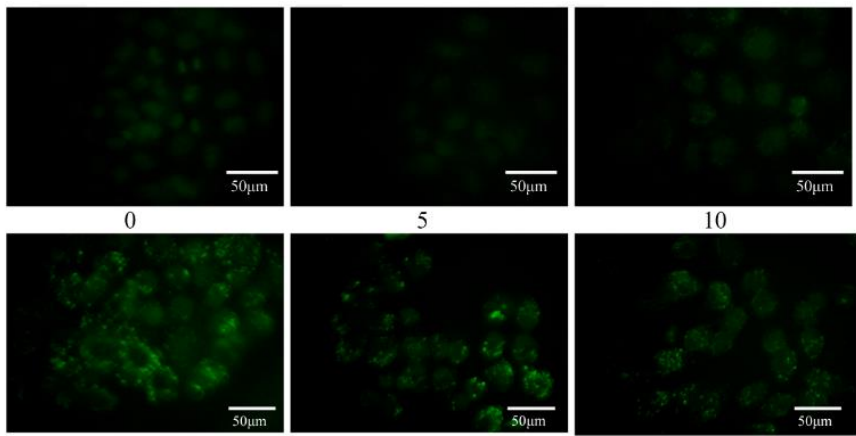

fluoxetine $(\mu \mathrm{M}$

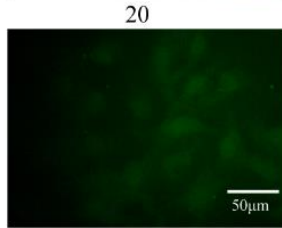

30

40

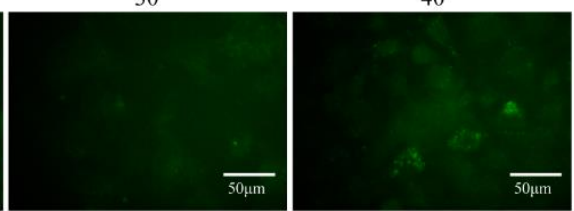

0

10

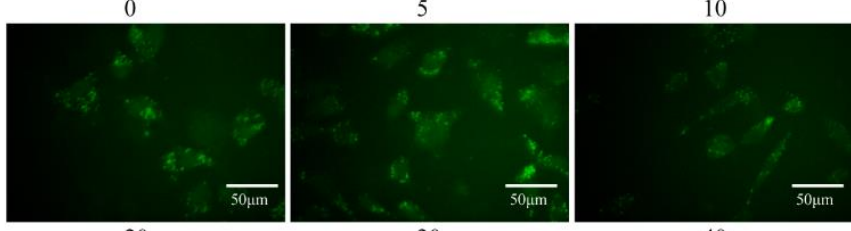

fluoxetine $(\mu \mathrm{M})$

20

A549

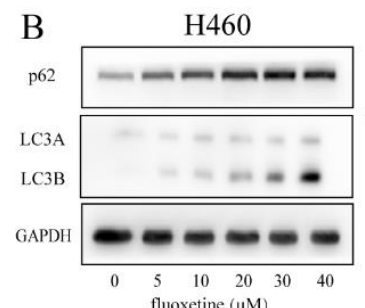

40

$\mathrm{C}$

$\mathrm{C}$

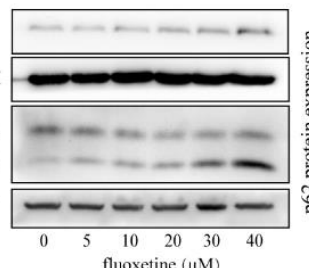

$\mathrm{H} 460$

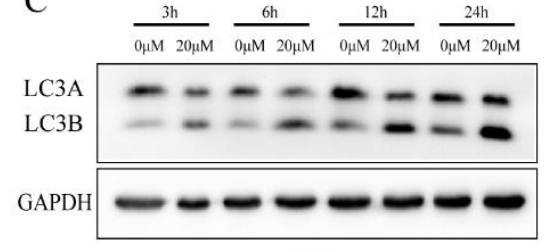

$\mathrm{A} 549$

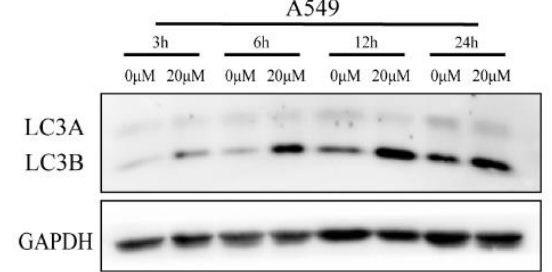

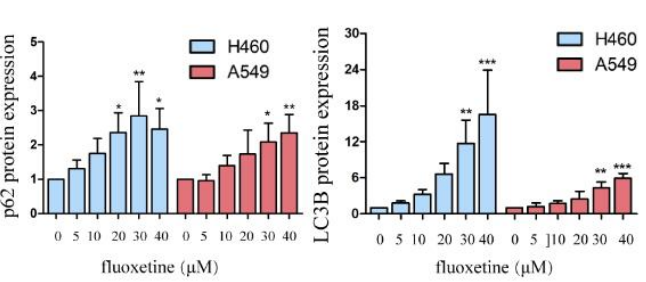

D
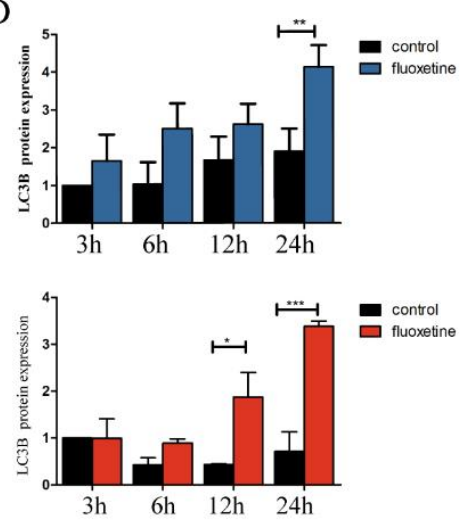

Figure.3: Fluoxetine induced autophagy in a dose and time-dependent way (A) The cells were treated with fluoxetine $(0-40 \mu \mathrm{M})$ for $24 \mathrm{~h}$. Immunofluorescence of LC3(green) was used to observe autophagy.

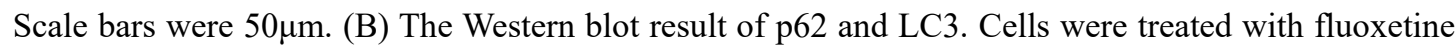
$(0-40 \mu \mathrm{M})$ for $24 \mathrm{~h}$. The images were collected from different parts of the same gel. (C) The Western blot result of LC3 in control and fluoxetine groups. Cells were treated with fluoxetine $(20 \mu \mathrm{M})$ for $3 \mathrm{~h}, 6 \mathrm{~h}, 12 \mathrm{~h}$, and 24h. The images were collected from different parts of the same gel. (D)Quantitative analysis of optical band densitometry. Data are presented as mean $\pm \mathrm{SD} ;{ }^{*} \mathrm{P}<0.05,{ }^{* *} \mathrm{P}<0.01,{ }^{* * *} \mathrm{P}<0.001$. 


\section{A}

A 1

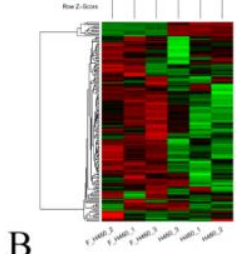

B

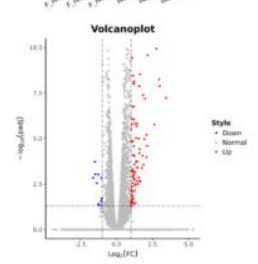

$\mathrm{C}$

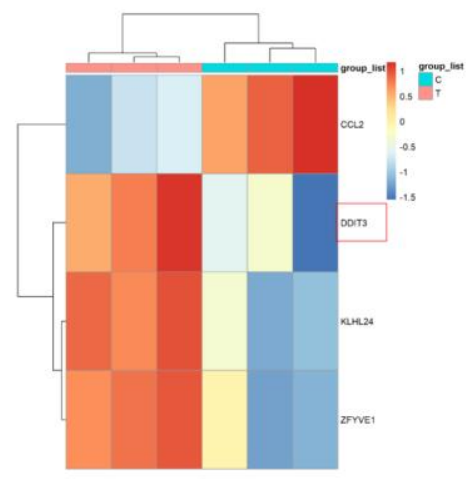

D

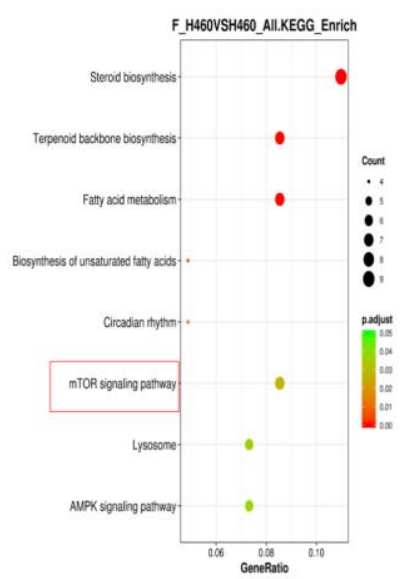

E
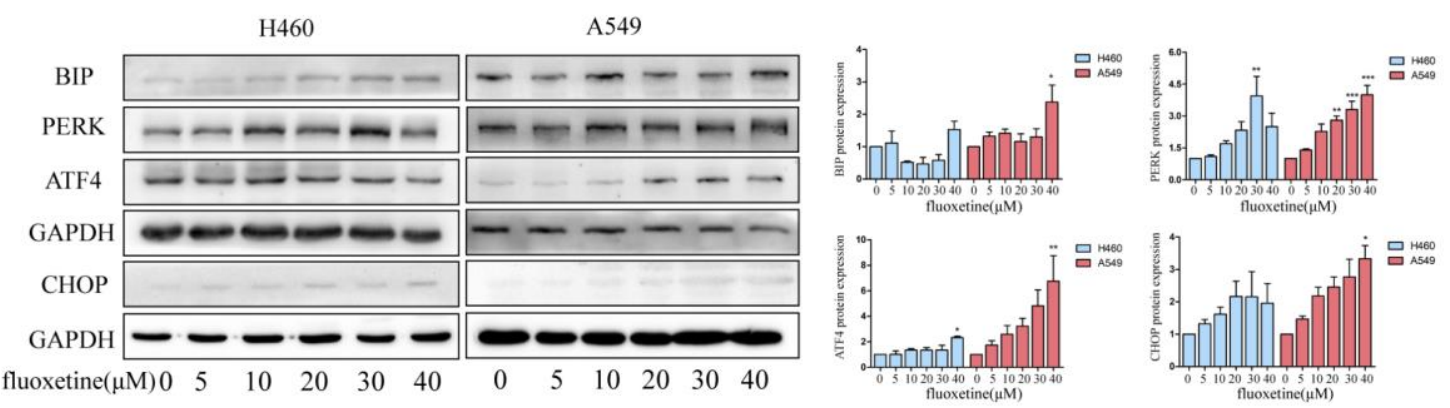

$\mathrm{F}$

H460

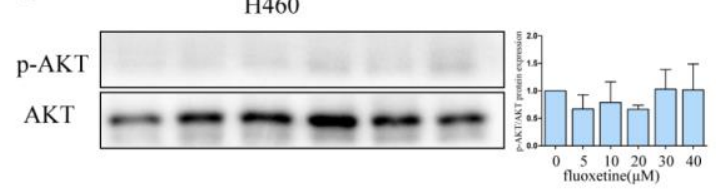

A549
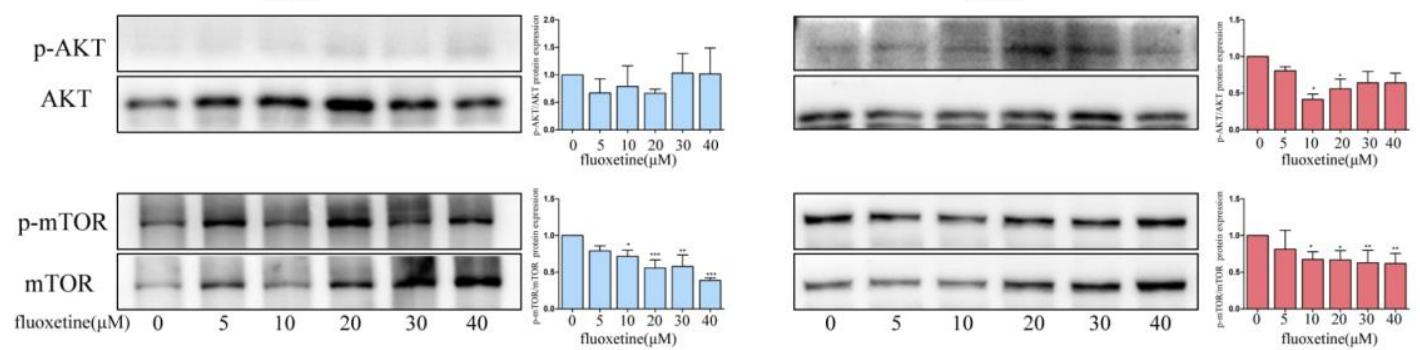

Figure.4: Fluoxetine had a connection with ER stress and mTOR signaling pathway (A) Heat map representing unsupervised hierarchical clustering of the expression level of mRNA in H460 cells treated with fluoxetine $(20 \mu \mathrm{M})$ for $24 \mathrm{~h}$. Each column represents the indicated sample. Each row represents one mRNA. Red and green indicate high and low expression, respectively. (B) The volcano plot represents 166 differentially regulated genes $(\mathrm{P}<0.05$, fold change $\geq 2)$. Red and blue indicate up and downregulation, respectively. (C) The autophagy-related genes were analyzed in a gene set. (D) The top 8 enriched KEGG pathways. (E)Western blot was used to analyze the expression ER stress related proteins, including BIP, PERK, ATF4, and CHOP. The images were collected from different parts of the same gel. (F) Western blot was used to analyze the expression of p-AKT, AKT, p-mTOR and mTOR. The images were collected from different parts of the same gel. Data are presented as mean $\pm \mathrm{SD} ; * \mathrm{P}<0.05$, **P $<0.01,{ }^{*} * * \mathrm{P}<0.001$. 
A

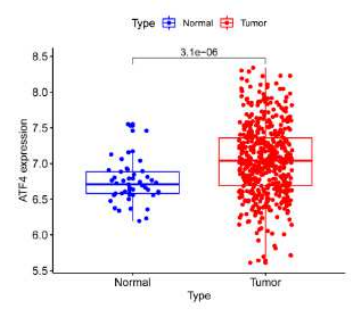

B

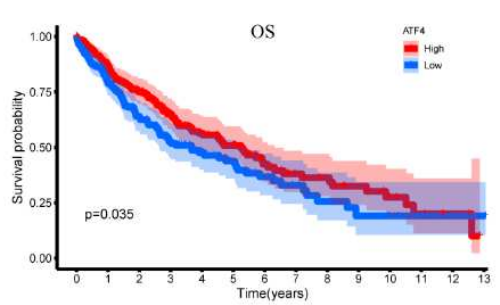

A549

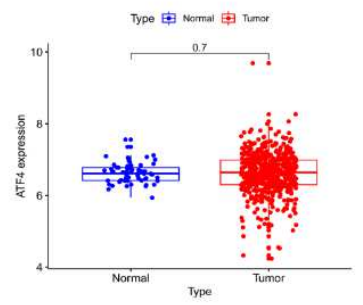

H460

ant

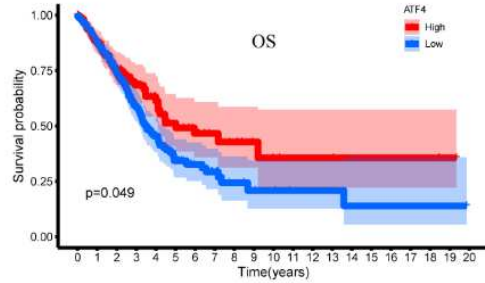

C
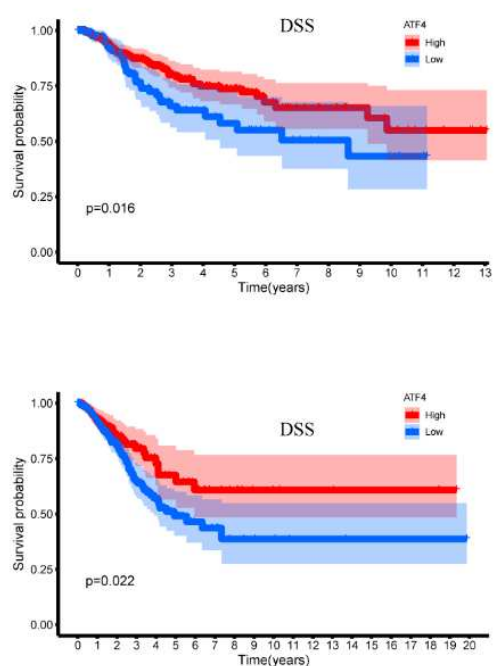

A549

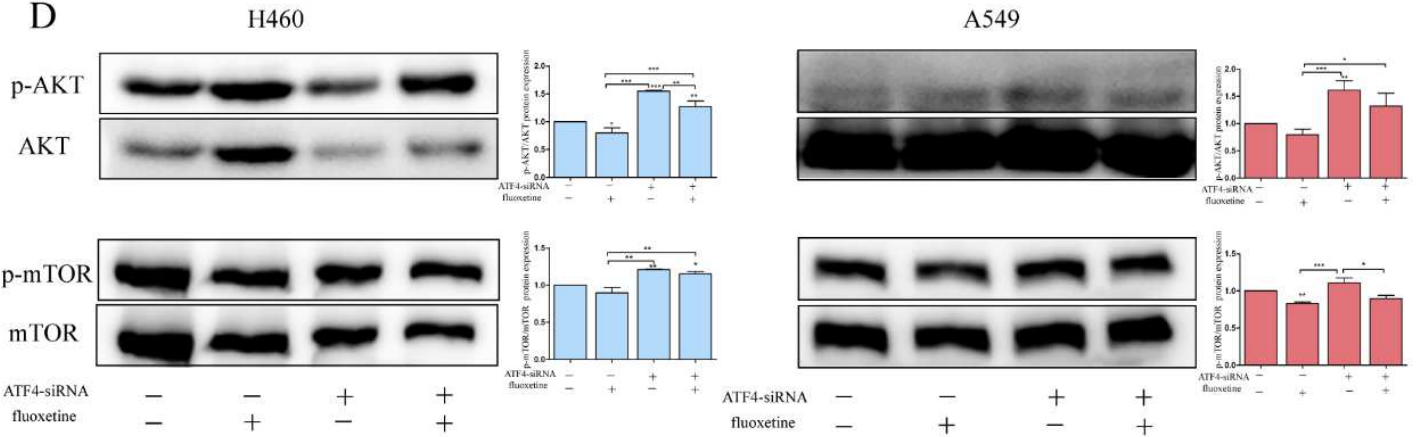

Figure.5: AKT/mTOR signaling pathway was regulated through ATF4 in fluoxetine treatment. (A) the expression of ATF4 in normal and tumor tissues was detected in the TCGA database. The blue spots on the left were the normal group, and the red spots on the right were the tumor group. (B-C) survival analysis comparing high to low expression of ATF4 in lung cancer. (D) the expression of p-AKT/AKT and $\mathrm{p}-\mathrm{mTOR} / \mathrm{mTOR}$ in lung cancer cells transfected with ATF4 siRNA for $48 \mathrm{~h}$ followed by exposure to fluoxetine $(20 \mu \mathrm{M})$ for another $24 \mathrm{~h}$ was tested by Western blot. The images were collected from different parts of the same gel. Data are presented as mean $\pm \mathrm{SD} ; * \mathrm{P}<0.05, * * \mathrm{P}<0.01, * * * \mathrm{P}<0.001$. 
A
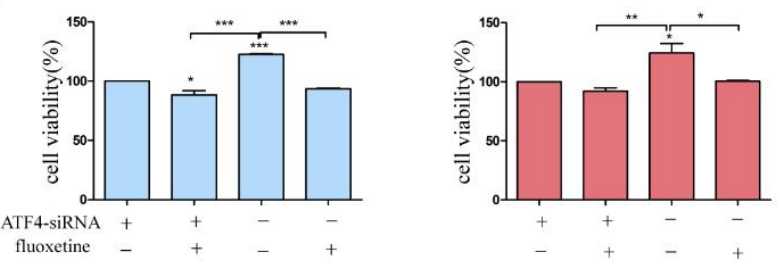

B

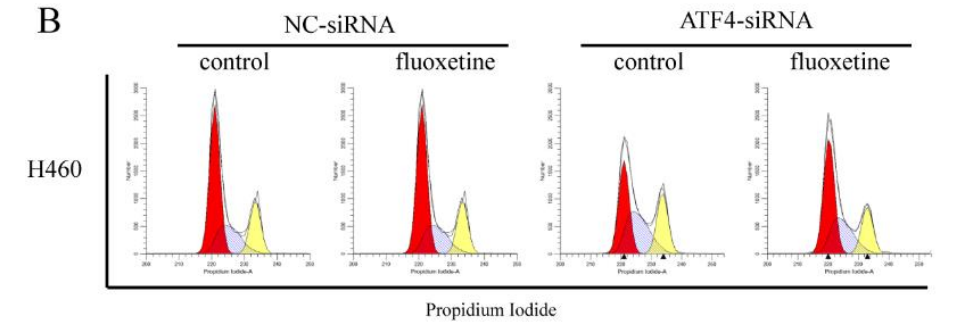

$\mathrm{C}$
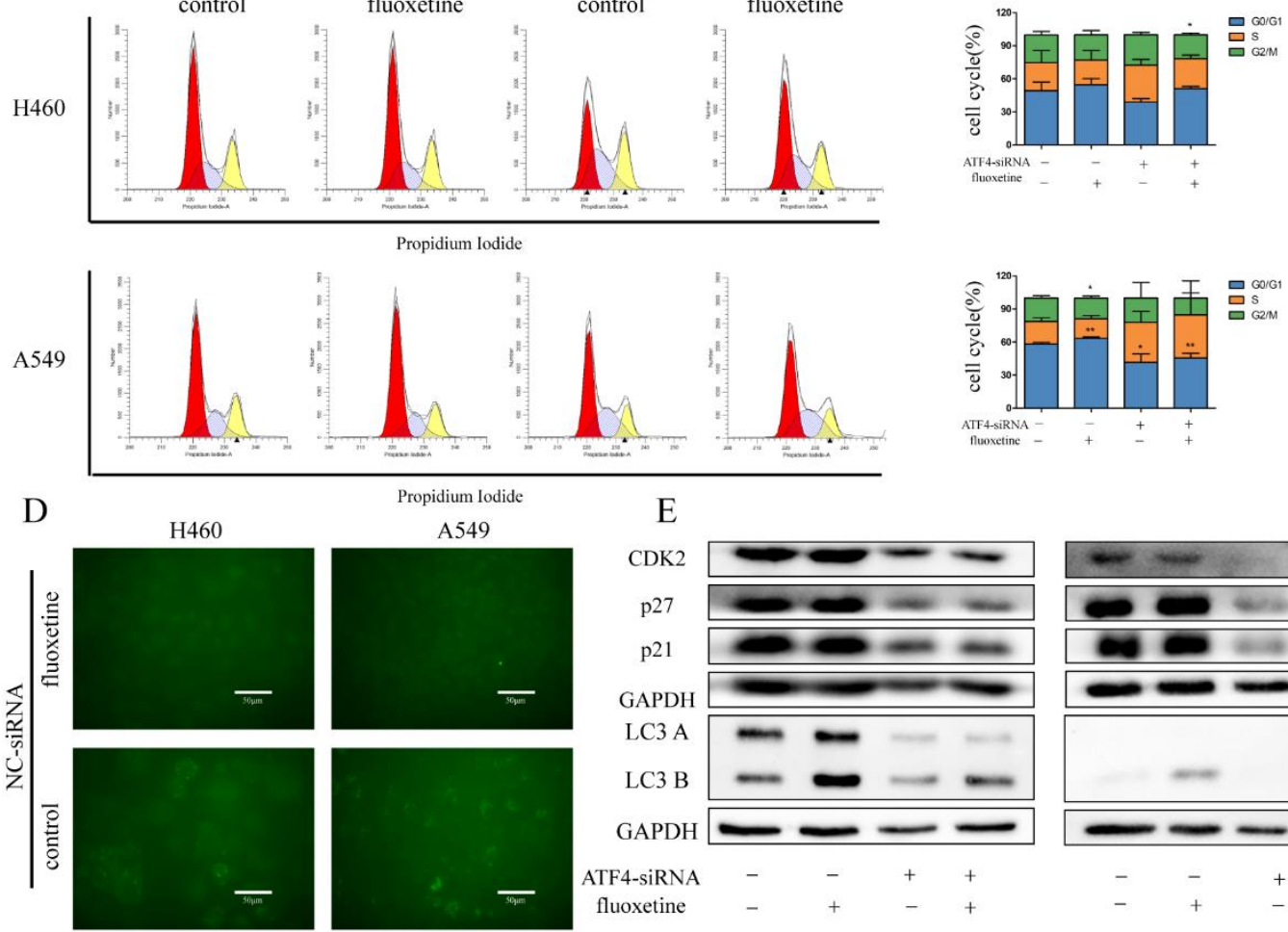

E
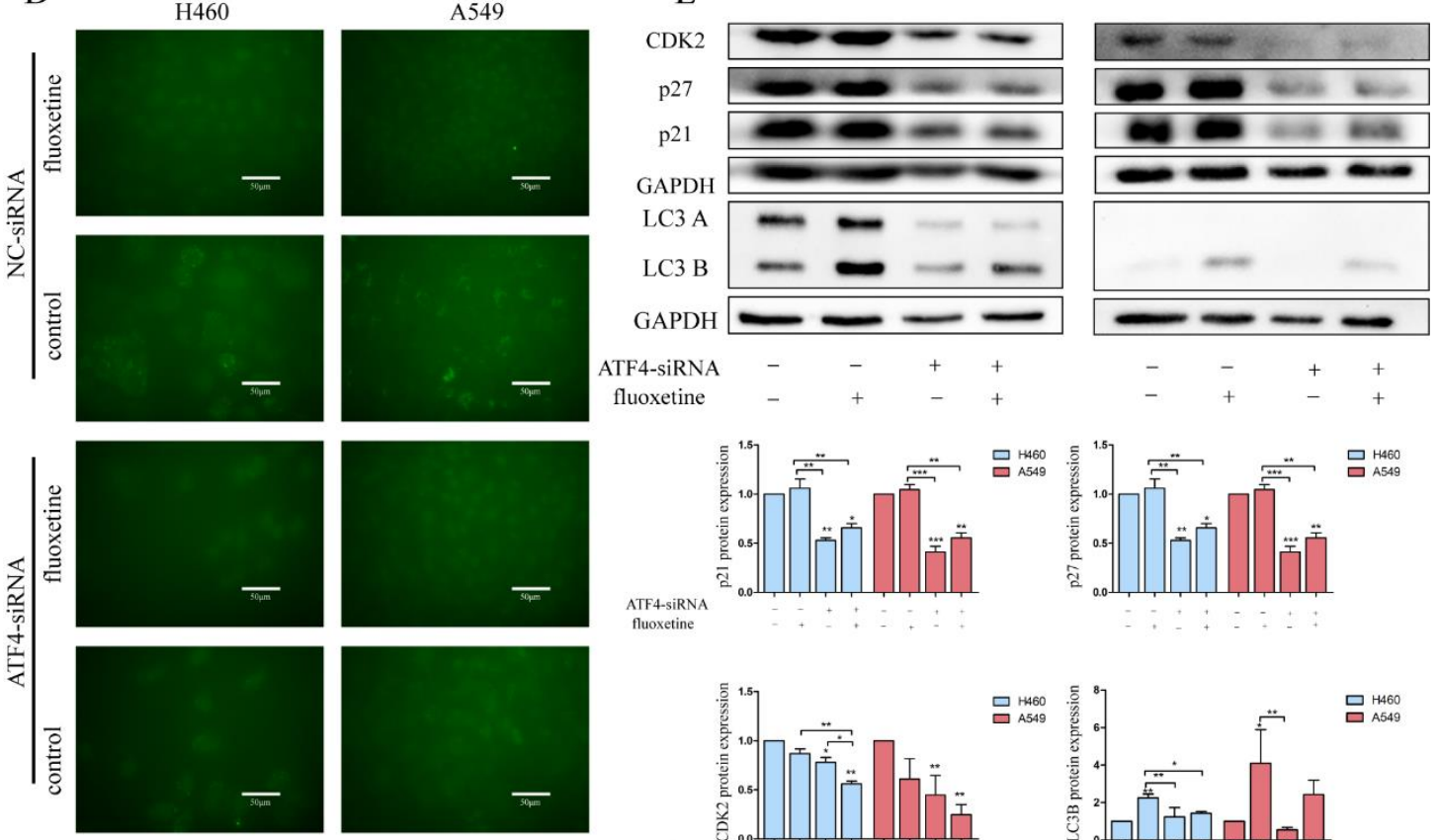

ATF4-siRNA
fluoxetine

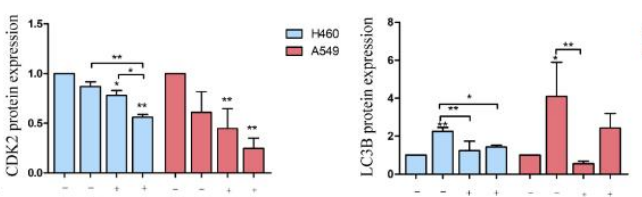

吕 ${ }_{\text {A HAs }}^{\text {Ast9 }}$

Figure.6: Fluoxetine-induced anticancer effect through the ATF4-AKT-mTOR signaling pathway (A) the cell viability in lung cancer cells transfected with ATF4 siRNA for $48 \mathrm{~h}$ followed by exposure to fluoxetine $(20 \mu \mathrm{M})$ for another $24 \mathrm{~h}$ was assessed by CCK8. Left and right indicate H460 cells and A549 cells, respectively. (B-C) the distribution of the cell cycle in lung cancer cells transfected with ATF4 siRNA for $48 \mathrm{~h}$ followed by exposure to fluoxetine $(20 \mu \mathrm{M})$ for another $24 \mathrm{~h}$ was analyzed by flow cytometry. (D) the autophagy in lung cancer cells transfected with ATF4 siRNA for $48 \mathrm{~h}$ followed by exposure to fluoxetine $(20 \mu \mathrm{M})$ for another $24 \mathrm{~h}$ was observed by immunofluorescence of LC3(green). Scale bars were $50 \mu \mathrm{m}$. (E) the expression of CDK2, p27, p21, and LC3 in lung cancer cells transfected with ATF4 siRNA for $48 \mathrm{~h}$ followed by exposure to fluoxetine $(20 \mu \mathrm{M})$ for another $24 \mathrm{~h}$ was tested by Western blot. Left and right indicate $\mathrm{H} 460$ cells and A549 cells, respectively. The images were 
collected from different parts of the same gel. Data are presented as mean $\pm \mathrm{SD} ; * \mathrm{P}<0.05, * * \mathrm{P}<0.01$, $* * * \mathrm{P}<0.001$.

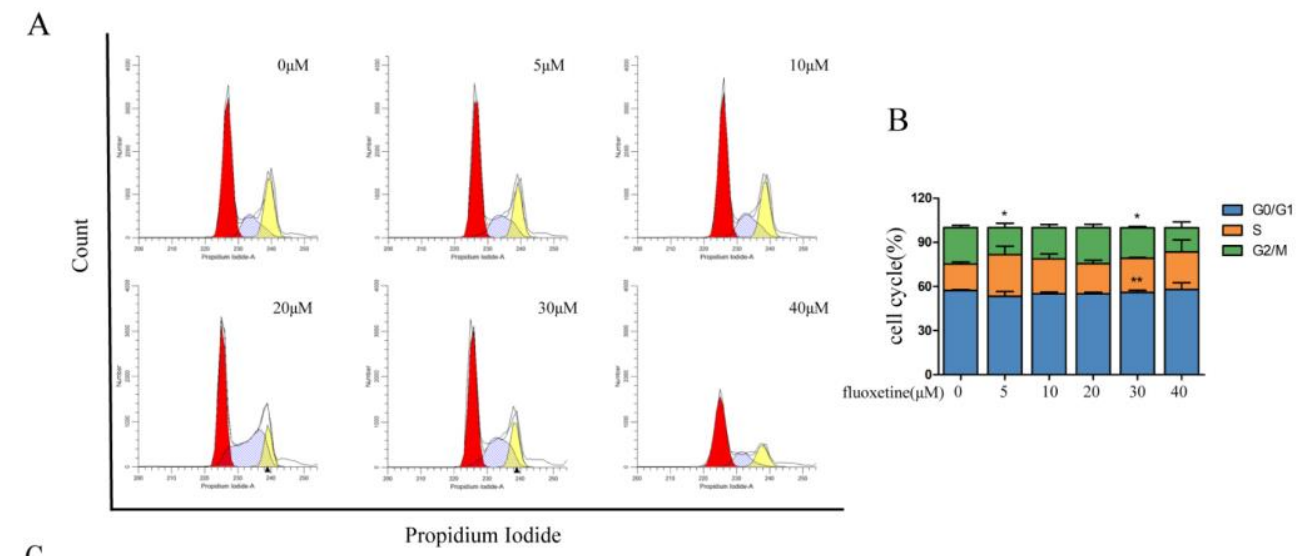

$$
\text { C }
$$

5

10

20

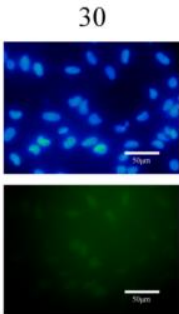

40
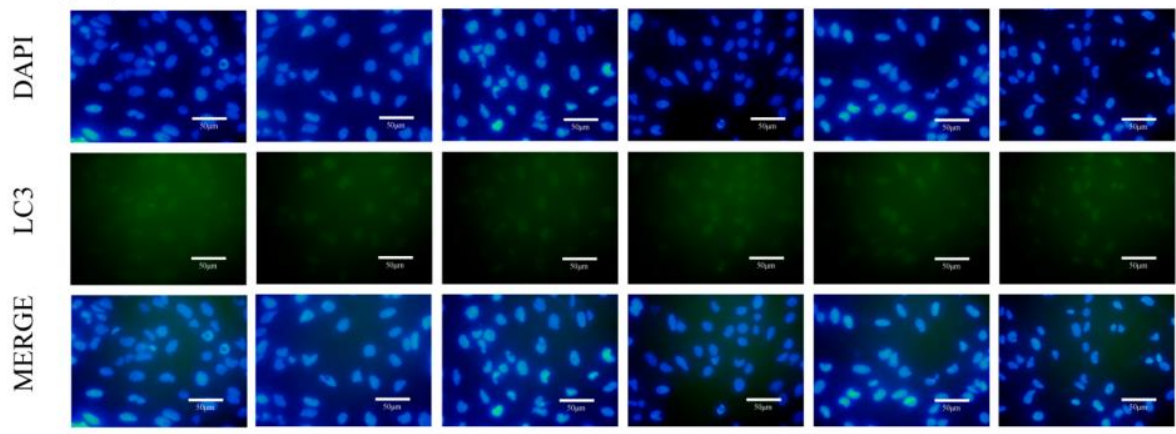

D

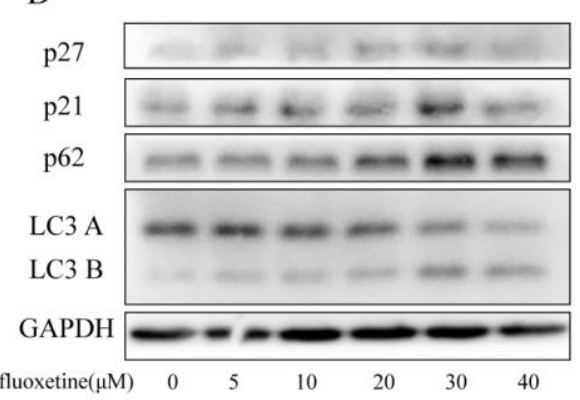

E

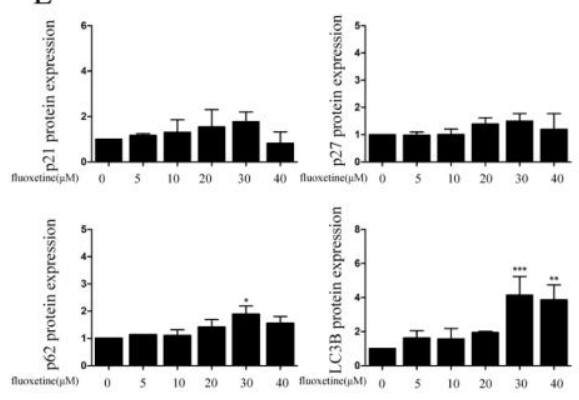

Figure.7: Fluoxetine exerted anti-tumor effects while not damaging normal cells. (A-B) the distribution of the cell cycle in normal lung epithelial cells treated with fluoxetine $(0-40 \mu \mathrm{M})$ was analyzed by flow cytometry. (C) immunofluorescence of LC3(green) and DAPI (blue) was used to observe the autophagy. Scale bars were $50 \mu \mathrm{m}$. (D-E) the expression of p27, p21, p62, and LC3 was tested by Western blot. The images were collected from different parts of the same gel. Data are presented as mean $\pm \mathrm{SD} ;{ }^{*} \mathrm{P}<0.05$, $* * \mathrm{P}<0.01, * * * \mathrm{P}<0.001$. 


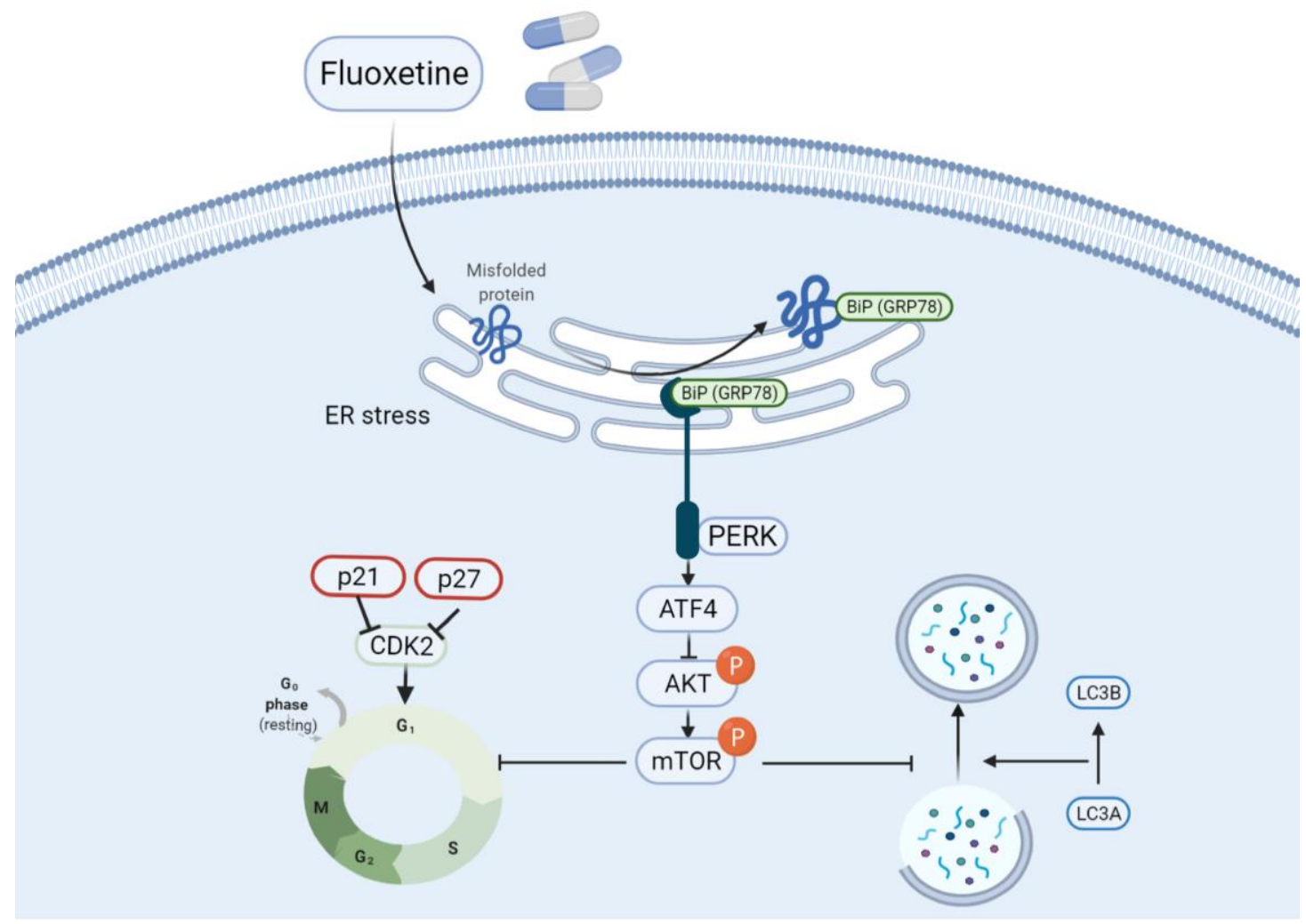

Figure.8: Fluoxetine induced cell cycle arrest and autophagy by triggering ATF4-AKT-mTOR signaling pathway in lung cancer cells. Briefly, fluoxetine activated the ER stress related proteins and the increased ATF4 inactivated the AKT/mTOR signaling pathway, thus leading to the cell cycle arrest at G0/G1 phase and autophagy. 


\section{Supplementary Files}

This is a list of supplementary files associated with this preprint. Click to download.

- supplementfileforScientificReports.pdf 